\title{
A post-HF approach \\ to the Sunscreen Octyl Methoxycinnamate
}

\author{
Ettore Fois, ${ }^{1}$ Mario Oriani, ${ }^{1}$ and Gloria Tabacchi ${ }^{1,}$ a) \\ Department of Science and High Technology, University of Insubria and INSTM UdR Insubria \\ Via Valleggio 11, I-22100 Como (Italy)
}

Octyl methoxycinnamate (2-ethylhexyl 4-methoxycinnamate, OMC) is a commercial sunscreen known as octinoxate with excellent UVB filter properties. However, it is known to undergo a series of photodegradation processes that decrease its effectiveness as UVB filter. In particular, the trans (E) form - which is considered so far the most stable isomer - converts to the cis $(\mathrm{Z})$ form under the effect of light. In this work, by using post-Hartree-Fock approaches $(\mathrm{CCSD}, \mathrm{CCSD}(\mathrm{t})$ and CCSD+T(CCSD)) on ground state OMC geometries optimized at the MP2 level we show that the cis and trans form of the gas-phase OMC molecule have comparable stability. Test calculations on the same structures with a series of dispersion-corrected DFT-based approaches including the B2PLYP double hybrid predict the trans structures to be energetically favored, missing the subtle stabilization of cis-OMC.

Our results suggest that the cis form is stabilized by intra-molecular dispersion interactions, leading to a folded, more compact structure than the trans isomer.

\section{INTRODUCTION}

The sunburn risk by UV has long been recognized: typically UVB radiation $(315 \mathrm{~nm}-280 \mathrm{~nm})$ induces erythema and direct DNA damage, while the deleterious consequence on human skin of UVA radiation (400 nm - $315 \mathrm{~nm})$ include, among others, DNA mutation and collagen degradations. ${ }^{1}$ The use of sunscreen products containing molecular UV filters is fundamental to prevent these harmful effects. ${ }^{2}$

Octyl methoxycinnamate (OMC) also known as 2ethylhexyl 4-methoxycinnamate, or by its commercial name octinoxate, is a widely used UVB filter in sunscreen products because it does not stain and does not cause skin irritation. ${ }^{2,3}$ In spite of its proven efficacy in UVB protection, various studies demonstrated that trans-octinoxate is unstable upon exposure to light, both in solution and in sunscreen lotions, leading to a decrease in photoprotection. ${ }^{4-19}$ Experiments conducted on sunscreen products indicated the predominance of octinoxate photolysis over photoisomerization due to interactions with other filters in the formulation. ${ }^{11,20,21}$ On the other hand, in the absence of other sunscreen agents, the trans-cis isomerization has been recognized as the prevailing photodegradation mechanism of octinoxate, both in protic solvents (methanol, water) and in apolar media such as cyclohexane. ${ }^{13,14}$ Both octinoxate isomers have very close maximum absorption wavelengths (about $290 \mathrm{~nm}$ in cyclohexane and around $310 \mathrm{~nm}$ in protic solvents). ${ }^{4,9-16,19}$ However, the cis form has a lower absorption capacity than the trans isomer, hence a lower efficacy as a UVB filter. ${ }^{2,10,13,14}$

More recently, experimental techniques such as ultrafast transient absorption spectroscopy, ${ }^{22,23}$ both in the gas phase and in microsolvation environments, have been adopted for evaluating the fate of cinnamate-based organic filters under UV irradiation. ${ }^{24-26}$ In particular, Tan et al. ${ }^{27}$, by employing resonance two-photon ionization (R2PI), examined the photorelaxation behaviour of trans-octinoxate as isolated

\footnotetext{
a)Electronic mail: gloria.tabacchi@uninsubria.it
}

molecules in the gas phase and in microsolvation water environment. They ascribed the measured nanosecond-scale lifetimes in the gas phase to a long-lived ${ }^{1} 1 n \pi^{*}$ state. Subsequent studies on other cinnamate derivatives evidenced that such a ${ }^{1} 1 n \pi^{*}$ state becomes destabilized in the presence of water, with a decrease of the relaxation lifetime to the order of ps. ${ }^{28,29}$ Peperstraete et al., by using time-resolved pump-probe spectroscopy, highlighted for gas-phase octinoxate a non-radiative decay from ${ }^{1} 1 \pi \pi^{*}$ to the ${ }^{1} 1 n \pi^{*}$ state and a subsequent deexcitation to the electronic ground state $\mathrm{S}_{0} \cdot{ }^{30}$ On the other hand, in solution environments (cyclohexane and ethanol) the trans-cis isomerization process becomes of greater relevance for the photochemistry of this molecule. ${ }^{30}$ Very recently, Muramatsu et al. ${ }^{31}$ adopted Resonant TwoPhoton Ionization (R2PI) and Laser-Induced-Fluorescence (LIF) to study the electronic spectrum of jet-cooled transoctinoxate. Using the UV/UV hole-burning technique and Density Functional Theory (DFT) calculations, these authors reported the existence of at least three isomers, which should be responsible of the broad feature observed in the R2PI spectra of jet-cooled octinoxate. ${ }^{27,31}$

In this context, a number of theoretical studies have investigated with a variety of techniques, including high-level quantum chemical approaches (complete active space selfconsistent field (CASSCF) and multi-state complete active space 2nd-order perturbation (MS-CASPT2)), the photophysical and photochemical properties of several cinnamate derivatives and/or reduced models of OMC - namely molecular structures including only the cinnamate chromophore nucleus but not the long aliphatic chain. ${ }^{32-43}$ For example, the trans-cis photoisomerization route of substituted methylcinnamates was recently explored via single-component artificial force-induced reaction (SC-AFIR) combined with timedependent density functional theory (TD-DFT), highlighting a marked influence of the phenyl substituents on the decay mechanism. ${ }^{42}$ Other studies at the TD-DFT/CIS level suggested appreciable solvent and hydrogen-bond effects on the photoisomerization mechanisms, ${ }^{28,29}$ which were then corroborated by CASSCF and MS-CASPT2 calculations. ${ }^{37,44}$ Overall, these researches on cinnamates basically agree on in- 
dicating $\mathrm{C}=\mathrm{C}$ bond twisting as the dominant reaction coordinate leading to a conical intersection, after which the molecule can either reconvert to the trans-form or evolve to the cisisomer. ${ }^{28,37,42,45,46}$ Nonetheless, the nature of the molecule in particular, the presence of phenyl ring substituents - may affect the relaxation dynamics in the excited states leading to different ratios of cis vs. trans-isomers. ${ }^{42,46}$ Also interestingly, whereas the photochemistry of the trans form of these cinnamate derivatives has been widely studied, less attention has been dedicated to the cis isomer - one exception being the model plant sunscreen methyl- ${ }^{41}$ and ethyl- ${ }^{47}$ sinapates, where the efficiency of photoprotection has been found to be independent of the trans or cis form. ${ }^{47}$

On a different note, other computational investigations were mostly devoted to the exploration of the stability of plausible isomers of the entire OMC molecule, and were performed at the DFT level. ${ }^{31,35,48-51}$ Indeed, a large number of conformational isomers can exist, due to the possible rotations of the propenyl single bond and the seven C-C bonds of the aliphatic chain. Overall, the latter studies indicated the predominant stability of the trans-OMC form over the cis-OMC form - in particular, an energy difference of $4.7 \mathrm{kcal} / \mathrm{mol}$ in favour of the trans isomer was calculated at the B3LYP/6$311++\mathrm{G}(\mathrm{d}, \mathrm{p})$ level. ${ }^{50}$ Regarding the trans-OMC, also a theoretical study of many possible conformers has been performed at the DFT level, indicating that several of these conformers are thermally accessible at ambient conditions, ${ }^{31}$ in line with the broad spectral patterns experimentally obtained. ${ }^{27,31}$

In this scenario, to the best of our knowledge, an investigation of the relative stability of cis- vs. trans- octinoxate beyond the DFT level of theory is still missing. Herein, we present a study based on post-Hartree-Fock (post-HF) calculations of different ground-state OMC forms, including three cis- (Z) and two trans- (E) isomers (see Fig. 1 for a graphical representation of the five structures). Because of the huge number of possible OMC conformers originated by single-bond rotation, and of the significantly higher CPU cost of post-HF vs. DFT-based methods, this study will not be focused on a thorough exploration of the extended conformational space of this molecule, but will be targeted at accurately studying a limited number of trans and cis representatives. Our aim is to identify, using correlated wavefunction methods, the leading features responsible of the trans vs cis stabilization.

\section{COMPUTATIONAL DETAILS}

A first guess structure for the trans-OMC conformer (Trans1) was obtained by annealing a first principles molecular dynamics (FPMD) ${ }^{52}$ trajectory (using the PBE-D2 functional ${ }^{53,54}$ ) from $300 \mathrm{~K}$ to $0 \mathrm{~K}$ in the gas phase. The structure of the second trans- conformer (Trans2) was taken from the literature - namely, the lowest-energy trans-conformer as reported in Ref. ${ }^{31}$, which was obtained at the $\omega \mathrm{b} 97 \mathrm{xd} / 6$ $311 \mathrm{~g}(\mathrm{~d}, \mathrm{p})$ level. ${ }^{31}$

As regards the cis forms of OMC, two cis-conformers (Cis 1 and Cis2) were selected in the same way as the Trans 1 conformer, by annealing two different FPMD trajectories from
$300 \mathrm{~K}$ to $0 \mathrm{~K}$. Since the resulting annealed geometries of both conformers were characterized by a packed structure (see Fig. 1), a cis-conformer (Cis3) with an unpacked structure was also searched, using as a guess geometry a cis-octinoxate model with a dihedral $\mathrm{O} 1=\mathrm{C} 2-\mathrm{C} 3=\mathrm{C} 4$ angle of 0 degrees (for atom labels, see Fig. 2).

The geometries of the above-described cis- and trans- OMC conformers were then optimized at the $\mathrm{mp} 2^{55}$ level of theory using the cc-pvdz basis set augmented by diffuse s-functions for all atoms $(\mathrm{mp} 2 / \mathrm{cc}-\mathrm{pvdz}+\mathrm{S})$. Also, normal modes analysis was performed at this level of theory in order to obtain zeropoint-energies (zpe). All frequencies were positive, thus the geometries were true minima in the potential energy surfaces. Adopting these optimized structures, single point mp2 energies (see Table I) were obtained by using larger basis sets, namely the Dunning-Hay full-double- $\zeta$ plus polarization plus diffuse dzp $++{ }^{56}$, the correlation-consistent basis sets aug-ccpvdz and aug-cc-pvtz, ${ }^{57}$ the triple- $\zeta$ plus polarization (tzp) and plus diffuse (tzp++). ${ }^{58,59}$ Table I comprises also the zpecorrected Hartree-Fock (HF) single-point energies calculated on the same geometries with the cc-pvdz+S and tzp++ basis set.

Moreover, single point energies of the five conformers were also calculated by adopting different Coupled-Clusters $(\mathrm{CC})^{60,61}$ approaches, namely the $\operatorname{CCSD}^{62}, \operatorname{CCSD}(\mathrm{t}),{ }^{63}$ and the CCSD-T(CCSD) ${ }^{64}$ approximations. In these cases, two different double- $\zeta$ basis set were used: the cc-pvdz-S one ${ }^{57}$, and a full double- $\zeta$ plus polarization and diffuse functions $(\mathrm{dzp}++)$. Zpe-corrected CC energies are reported in Table II. The Frozen Core approximation was adopted throughout, with the only exception of the tzp++ basis set.

To get a deeper insight on how post-HF energetics would compare with DFT-based results on this molecule, we also performed a series of single point calculations on the MP2/ccpvdz + S optimized geometries of the five conformers with density functional approximations belonging to different rungs of the Perdew's "Jacob's Ladder" ${ }^{65}$ Specifically, besides PBE (the "rung-2" GGA functional used in the exploratory FPMD simulations), we considered the "rung-4" range-separated hybrid functionals $\omega \mathrm{b} 97 \mathrm{xd}^{66}$ and LC- $\omega$ PBE. ${ }^{67}$ Moreover, as representative of the highest rung of the ladder, the double hybrid B2PLYP68 ("rung-5") was selected. Both the PBE and B2PLYP functionals were complemented by D3BJ empirical dispersion, with Becke-Johnson damping. ${ }^{69}$ These functionals were tested with the same basis sets used for the mp2 calculations.

The FPMD runs for the guess structures were performed using the CPMD code, ${ }^{52,70,71}$ adopting ultra-soft pseudopotentials at 30 Ry cutoff of the wavefunctions expansion with planewave basis set, while the rest of the adopted FPMD protocol was as in Refs. ${ }^{72-77}$. The single-point energy calculations on the optimized MP2/cc-pvdz-S geometries of the five conformers were performed using the same energy cutoff and an isolated ${ }^{78}$ cubic box of $100 \times 100 \times 100$ bohr $^{3}$ size.

The geometry optimization and mp2 calculations on the guess structures were performed with the Gaussian0979 code, while all the $\mathrm{CC}$ calculations were performed with the NWCHEM ${ }^{80,81}$ code. The $\omega b 97 x d$, LC- $\omega$ PBE and B2PLYP 

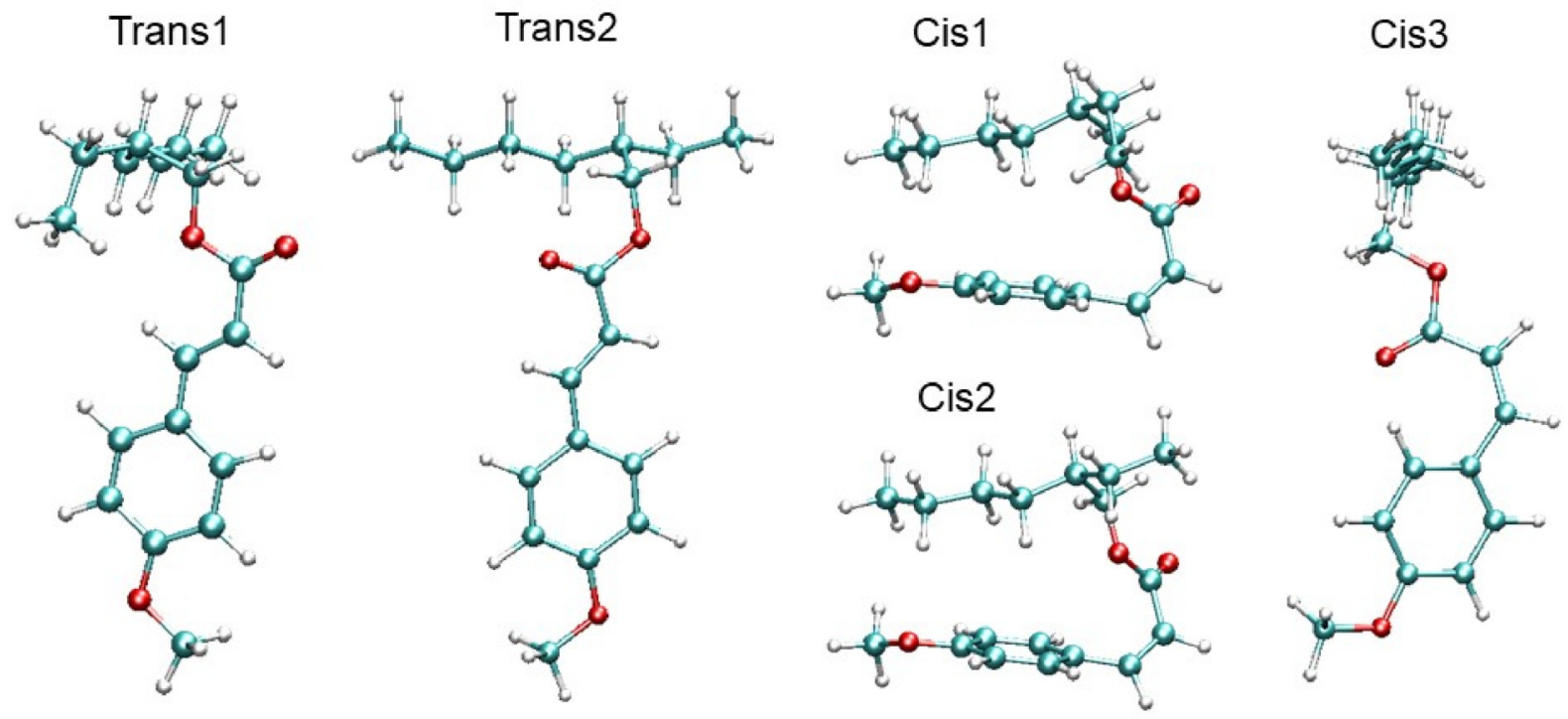

FIG. 1. Graphical representation of the mp2/cc-pvdz+S optimized structures of the five selected OMC conformers. Atom color codes: $\mathrm{O}=\mathrm{red}$; $\mathrm{C}=$ cyan; $\mathrm{H}=$ white.

calculations were performed with Gaussian 09. Basis sets were available either from Gaussian09/NWCHEM or from the Basis Set Exchange library at basissetexchange.org. ${ }^{82}$

\section{RESULTS AND DISCUSSION}

The five optimized structures, depicted in Fig. 1, suggest the leitmotiv underlining the relative stability of the different forms of OMC. In the trans-OMC structures, the extension of the $\pi$ conjugation seems to be the stabilizing and structuredetermining driving force. A similar conclusion can be drawn for the Cis3 isomer, which has a roughly planar geometry and manifests a certain degree of $\pi$ conjugation, as evidenced by the $\mathrm{C} 2-\mathrm{C} 3$ and $\mathrm{C} 4-\mathrm{C} 5$ bond distances, which are closer to the values exhibited by the trans isomers. On the other hand, in the Cis 1 and Cis 2 forms of OMC, the van-der-Waals like interaction ( $\mathrm{vdw}$ ) between the cinnamate backbone and the aliphatic chain appears to be the structure-determining driving force.

Interestingly, the two kinds of interaction, the $\pi$ conjugation vs vdw, seem to be competitive and reciprocally limiting. Whereas the small number of studied conformers surely does not exhaust the possible relative arrangements of the aliphatic chain and the cinnamate backbone in OMC, the inclusion in our set of an approximately planar cis structure (which should have an appreciable conjugation but tiny intramolecular dispersion) along with highly packed cis-isomers allows us to investigate on a quantitative and more general basis the competition between $\pi$-conjugation and vdw effects.

The relevant dihedral angles involving the $\mathrm{Z}$ or $\mathrm{E} \mathrm{C}=\mathrm{C}$ dou- ble bond are reported in Fig. 2. In the Cis1 and Cis2 isomers, these dihedrals - in particular $\mathrm{O} 1=\mathrm{C} 2-\mathrm{C} 3=\mathrm{C} 4-$ clearly indicate large distortions of the cinnamate backbone from the ideal planar structure, which would be functional to a stabilizing $\pi$-conjugation. Conversely, in the trans- forms, the cinnamate out-of-plane distortion is quite modest. However, the $\mathrm{E}$ arrangement of the $\mathrm{C} 3=\mathrm{C} 4$ double bond (see Fig. 2) does not allow for the intra-molecular vdw stabilizing interaction envisaged in the cis-OMC structures. Besides the dihedral angles values - that highlight a more planar conjugate backbone - also the C4-C5 distances, which are shorter in both trans conformers, indicate that the trans conformers have a more extended $\pi$ structure than the cis ones. As regards Cis3, the $\mathrm{C} 2-\mathrm{C} 3=\mathrm{C} 4-\mathrm{C} 5$ dihedral angle slightly deviates from planarity $\left(-5.4^{\circ}\right)$, preventing $\mathrm{Cis} 3$ to reach the conjugation extent of the trans forms. Nonetheless, Cis 3 seems more similar to the trans conformers rather than to the Cis $1 /$ Cis 2 ones. Therefore, it is expectable that intra-molecular vdw stabilization would not play a relevant role in $\mathrm{Cis} 3$.

Actually, the selected set of conformers poses the challenging issue of whether intra-molecular vdw interactions might compete with extended $\pi$ conjugation. Importantly, such an issue has practical relevance as well, because both trans-to-cis and cis-to-trans light-induced isomerizations are supposed to occur in commercial OMC-based sunscreen products.

Let us now discuss the energetics of the studied OMC conformers. First of all, we notice that the zpe is higher for the cis isomers characterized by a packed structure (Cis1/Cis2), suggesting that conformers having an unpacked geometry might be slightly favoured in this respect. Actually, this occurs at the mp2/cc-pvdz+S level, where the Cis2 electronic energy (924.4519260 Hartree) is lower than the corresponding value for the Trans 2 conformer (-924.4514416 Hartree) - amount- 


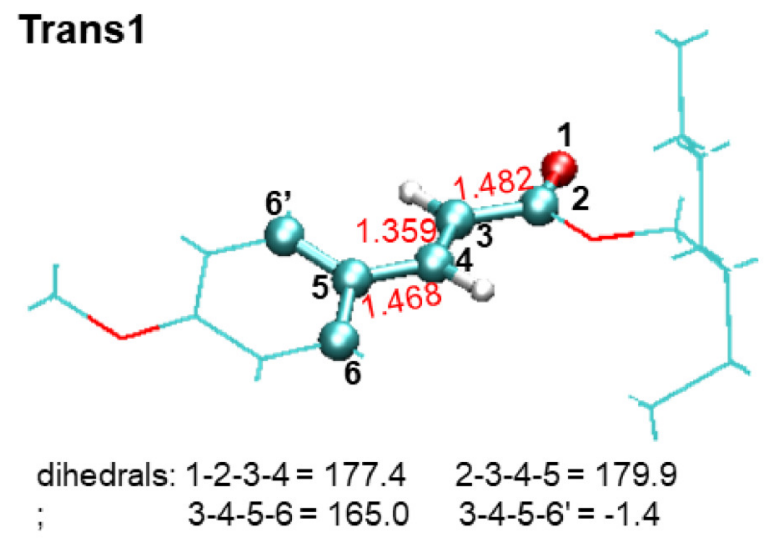

Cis1

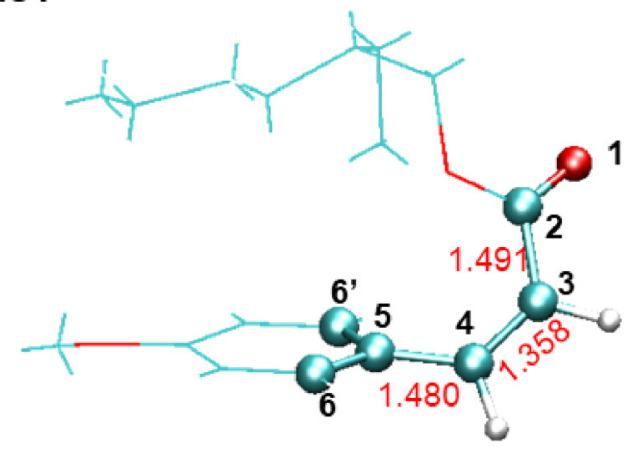

dihedrals: $1-2-3-4=-152.7 \quad 2-3-4-5=8.3$ $3-4-5-6=-131.7 \quad 3-4-5-6^{\prime}=53.2$
Trans2

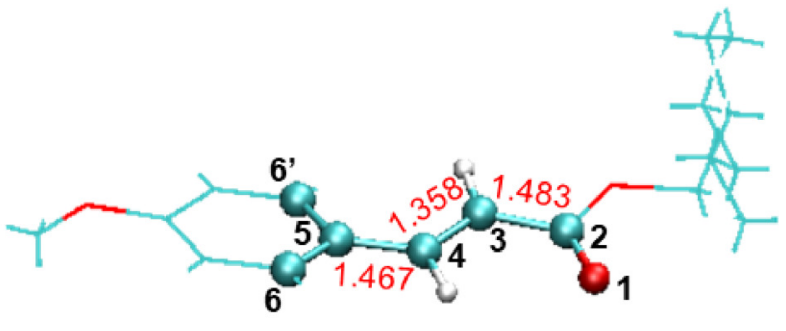

dihedrals: $\quad 1-2-3-4=1.4 \quad 2-3-4-5=179.7$

$3-4-5-6=12.8 \quad 3-4-5-6^{\prime}=-179.9$

\section{Cis2}

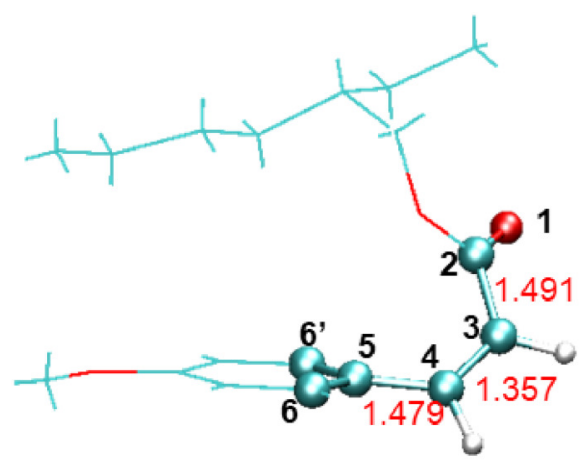

dihedrals: $\quad 1-2-3-4=-144.5 \quad 2-3-4-5=7.4$

$3-4-5-6=-135.2 \quad 3-4-5-6=35.3$

\section{Cis3}

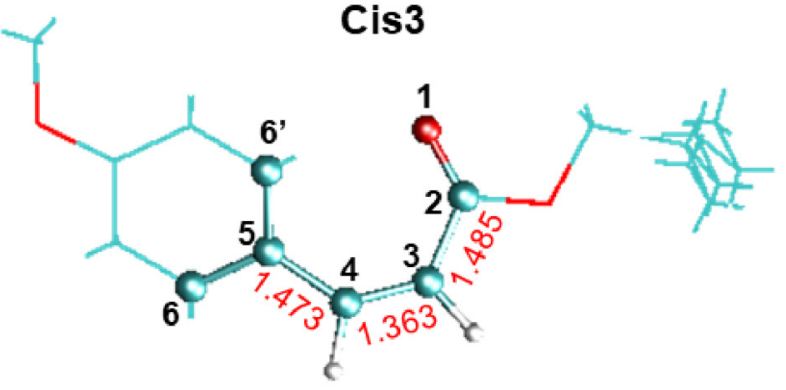

dihedrals: $1-2-3-4=-2.4$

$2-3-4-5=-5.4$

$3-4-5-6=150.6$

$$
3-4-5-6^{\prime}=-33.8
$$

FIG. 2. Graphical representation of the five mp2/cc-pvdz+S optimized structures of OMC. Atoms relevant for $\pi$ conjugation are represented as ball-and-sticks, while the others are represented as lines (color codes as in Fig.1). Angles in degrees, distances in $\AA$.

ing to an electronic energy difference of $-0.304 \mathrm{kcal} / \mathrm{mol}$ in favor of Cis2. Upon addition of the zpe, Cis2 becomes less stable than Trans 2 by $0.223 \mathrm{kcal} / \mathrm{mol}$ (Table I). Interestingly, at the same level of theory, the zpe contribution also reverts the stability order of the Cis1 and Trans1 forms - namely, the Cis 1 vs Trans 1 electronic energy difference is $-0.915 \mathrm{kcal} / \mathrm{mol}$ in favour of Cis1. These results may appear unexpected, as, in the previous literature, on the basis of hybrid-DFT calcula- tions, OMC trans isomers (with respect to the $\mathrm{C} 3=\mathrm{C} 4$ double bond) are always quoted to be more stable than the cis ones in both gas and solution phases.$^{48,50,83}$

On the other hand, the higher stability of Trans 2 with respect to Trans1 is not surprising, as the Trans 2 geometry had been obtained from an accurate conformational analysis refined at the DFT level, including both approximated dispersion and zpe corrections. ${ }^{31}$ Indeed, the Trans 2 structure is 
TABLE I. Zpe and zpe-corrected MP2 energies (Hartree) and relative energies $\Delta E(\mathrm{kcal} / \mathrm{mol})$ of the five conformers with different basis sets.

\begin{tabular}{|c|c|c|c|c|c|}
\hline Basis set ${ }^{\mathrm{a}}$ & Cis1 & Trans1 & Cis2 & Trans2 & Cis 3 \\
\hline zpe-mp2/cc-pvdz+S & 0.410052 & 0.409026 & 0.409977 & 0.409137 & 0.409217 \\
\hline cc-pvdz+S & -924.040302 & -924.039870 & -924.041949 & -924.042305 & -924.034289 \\
\hline$\Delta E$ & 1.257 & 1.528 & 0.223 & 0.000 & 5.030 \\
\hline dzp++ & -924.144505 & -924.141180 & -924.145834 & -924.143490 & -924.135897 \\
\hline$\Delta E$ & -0.637 & 1.449 & -1.471 & 0.000 & 4.765 \\
\hline aug-cc-pvdz & -924.197677 & -924.193647 & -924.198970 & -924.196188 & -924.187801 \\
\hline$\Delta E$ & -0.934 & 1.595 & -1.746 & 0.000 & 5.263 \\
\hline aug-cc-pvtz & -925.008744 & -925.010046 & -925.010656 & -925.013037 & -925.004292 \\
\hline$\Delta E$ & 2.694 & 1.877 & 1.494 & 0.000 & 5.488 \\
\hline tzp & -924.924976 & -924.927379 & -924.926980 & -924.930495 & -924.921723 \\
\hline$\Delta E$ & 3.464 & 1.955 & 2.206 & 0.000 & 5.505 \\
\hline tzp++ & -925.000783 & -925.001053 & -925.002618 & -925.004079 & -924.995208 \\
\hline$\Delta E$ & 2.068 & 1.899 & 0.917 & 0.000 & 5.567 \\
\hline tzp++ (Full Core) & -925.430020 & -925.428168 & -925.431526 & -925.431100 & -925.422146 \\
\hline$\Delta E$ & 0.678 & 1.840 & -0.267 & 0.000 & 5.619 \\
\hline $\mathrm{HF} / \mathrm{cc}-\mathrm{pvdz}+\mathrm{S}$ & -920.995261 & -921.014042 & -920.999511 & -921.016629 & -921.008522 \\
\hline$\Delta E$ & 13.408 & 1.623 & 10.741 & 0.000 & 5.087 \\
\hline $\mathrm{HF} / \mathrm{tzp}++$ & -921.229814 & -921.251164 & -921.234532 & -921.253758 & -921.239166 \\
\hline$\Delta E$ & 15.025 & 1.628 & 12.065 & 0.000 & 9.157 \\
\hline
\end{tabular}

${ }^{a}$ Zpe-corrections and geometries were obtained by optimizing all conformers at the MP2/cc-pvdz+S level. The last four lines refer to HF calculations on the same geometries. $\Delta E$ s are relative to the Trans 2 energies.

TABLE II. Zpe-corrected Coupled Cluster energies (Hartree) and relative energies $\Delta E(\mathrm{kcal} / \mathrm{mol})$ of the five conformers with different basis sets.

\begin{tabular}{|c|c|c|c|c|c|}
\hline Basis set $^{\mathrm{a}}$ & Cis 1 & Trans1 & Cis2 & Trans 2 & Cis3 \\
\hline CCSD/cc-pvdz+S & -924.203270 & -924.207112 & -924.205486 & -924.209219 & -924.201682 \\
\hline$\Delta E$ & 3.733 & 1.322 & 2.342 & 0.000 & 4.730 \\
\hline $\operatorname{CCSD}(t) / c c-p v d z+S$ & -924.326933 & -924.328537 & -924.328767 & -924.330864 & -924.323221 \\
\hline$\Delta E$ & 2.466 & 1.460 & 1.316 & 0.000 & 4.796 \\
\hline $\mathrm{CCSD}+\mathrm{T}(\mathrm{CCSD}) / \mathrm{cc}-\mathrm{pvdz}+\mathrm{S}$ & -924.331918 & -924.333471 & -924.333756 & -924.335955 & -924.328242 \\
\hline$\Delta E$ & 2.533 & 1.559 & 1.380 & 0.000 & 4.840 \\
\hline $\mathrm{CCSD} / \mathrm{dzp}++$ & -924.302624 & -924.303659 & -924.304522 & -924.305618 & -924.298455 \\
\hline$\Delta E$ & 1.879 & 1.230 & 0.688 & 0.000 & 4.495 \\
\hline $\operatorname{CCSD}(\mathrm{t}) / \mathrm{dzp}++$ & -924.424804 & -924.423100 & -924.426275 & -924.425232 & -924.418052 \\
\hline$\Delta E$ & 0.269 & 1.338 & -0.654 & 0.000 & 4.506 \\
\hline $\mathrm{CCSD}+\mathrm{T}(\mathrm{CCSD}) / \mathrm{dzp}++$ & -924.430358 & -924.428582 & -924.431827 & -924.430874 & -924.423631 \\
\hline$\Delta E$ & 0.324 & 1.438 & -0.598 & 0.000 & 4.545 \\
\hline
\end{tabular}

${ }^{a}$ Zpe corrections and geometries were obtained by optimizing all conformers at the MP2/cc-pvdz+S level. $\Delta E$ s are relative to the Trans2 energies.

TABLE III. Moments of Inertia (a.u.)

\begin{tabular}{crrr}
\hline \hline & & & \\
\hline Cis1 & 7377.9281 & 4871.3189 & 9168.6493 \\
Trans1 & 15941.9319 & 4225.6962 & 16912.0170 \\
Cis2 & 7629.6061 & 4904.8131 & 10007.9806 \\
Trans2 & 17820.7804 & 3793.3202 & 20231.0798 \\
Cis3 & 17156.4061 & 3902.7448 & 18943.8021 \\
\hline \hline
\end{tabular}

characterized by a very good planarity, as also evidenced by the dihedral angles and by the C4-C5 bond length, suggesting a stronger degree of $\pi$ delocalization between the cinnamate phenyl ring and the (E)-C3=C4 double bond (see Fig. 2). As regards the energetics of the cis structures, a possible explanation of the greater stability of Cis 2 vs Cis 1 is the nearly-linear zig-zag arrangement of the alkyl chain, along with the higher extent of $\pi$ conjugation as denoted by the slightly shorter C4-C5 bond length. Finally, both Cis1 and Cis2 are significantly more stable than Cis3, which has an electronic energy of -924.443506 Hartrees - that is, 5.284 and $4.297 \mathrm{kcal} / \mathrm{mol}$ greater than Cis2 and Cis1, respectively. Hence the addition of the zpe contribution does not change the stability order of the three cis forms. Indeed, Cis3 is the highest energy conformer among the structures here considered: it has a lower $\pi$-conjugation than the trans form, and at the same time it lacks the substantial dispersion stabilization of the packed cis structures. 
TABLE IV. Zpe-corrected relative energies $\Delta E(\mathrm{kcal} / \mathrm{mol})$ of the five conformers with different DFT approximations and basis sets.

\begin{tabular}{|c|c|c|c|c|c|}
\hline Level of theory ${ }^{\mathrm{a}}$ & Cis1 & Trans1 & Cis2 & Trans2 & Cis 3 \\
\hline PBE/PW & 13.039 & 2.869 & 10.671 & 0.000 & 5.390 \\
\hline PBE-D2/PW & 7.323 & 2.769 & 5.918 & 0.000 & 5.923 \\
\hline PBE-D3BJ/PW & 7.603 & 2.551 & 6.140 & 0.000 & 5.670 \\
\hline PBE-D3BJ/cc-pvdz+S & 5.700 & 2.652 & 4.547 & 0.000 & 5.880 \\
\hline LC- $\omega$ PBE-D3BJ/cc-pvdz+S & 3.428 & 2.056 & 2.318 & 0.000 & 5.599 \\
\hline$\omega b 97 x d / c c-p v d z+S$ & 3.254 & 2.014 & 2.466 & 0.000 & 5.652 \\
\hline$\omega \mathrm{b} 97 \mathrm{xd} / \mathrm{dzp}++$ & 4.306 & 1.794 & 3.380 & 0.000 & 5.503 \\
\hline$\omega b 97 x d / a u g-c c-p v d z$ & 3.279 & 1.718 & 2.489 & 0.000 & 5.660 \\
\hline$\omega \mathrm{b} 97 \mathrm{xd} / \mathrm{tzp}$ & 4.552 & 2.006 & 3.564 & 0.000 & 5.752 \\
\hline$\omega \mathrm{b} 97 x d / t z p++$ & 4.482 & 1.903 & 3.421 & 0.000 & 5.634 \\
\hline B2PLYP/dzp++ & 8.648 & 2.127 & 6.822 & 0.000 & 4.981 \\
\hline B2PLYP-D3BJ/dzp++ & 4.535 & 1.911 & 3.399 & 0.000 & 5.177 \\
\hline B2PLYP/cc-pvdz+S & 8.500 & 2.312 & 6.656 & 0.000 & 5.215 \\
\hline B2PLYP-D2/cc-pvdz+S & 4.307 & 2.238 & 3.169 & 0.000 & 5.606 \\
\hline B2PLYP-D3BJ/cc-pvdz+S & 4.410 & 2.090 & 3.243 & 0.000 & 5.422 \\
\hline B2PLYP/aug-cc-pvdz & 8.262 & 2.081 & 6.445 & 0.000 & 5.347 \\
\hline B2PLYP-D2/aug-cc-pvdz & 4.069 & 2.008 & 2.958 & 0.000 & 5.738 \\
\hline B2PLYP-D3BJ/aug-cc-pvdz & 4.148 & 1.865 & 3.022 & 0.000 & 5.542 \\
\hline B2PLYP/aug-cc-pvtz & 10.152 & 2.280 & 8.083 & 0.000 & 5.247 \\
\hline B2PLYP-D3BJ/aug-cc-pvtz & 6.039 & 2.064 & 4.661 & 0.000 & 5.443 \\
\hline B2PLYP/tzp & 10.376 & 2.378 & 8.314 & 0.000 & 5.343 \\
\hline B2PLYP-D3BJ/tzp & 6.263 & 2.162 & 4.891 & 0.000 & 5.539 \\
\hline B2PLYP/tzp++ & 9.871 & 2.287 & 7.810 & 0.000 & 5.289 \\
\hline B2PLYP-D3BJ/tzp++ & 5.757 & 2.071 & 4.387 & 0.000 & 5.484 \\
\hline $\mathrm{HF} / \mathrm{tzp}++^{\mathrm{b}}$ & 15.025 & 1.628 & 12.065 & 0.000 & 9.157 \\
\hline
\end{tabular}

${ }^{\text {a }} \mathrm{Zpe}$ corrections and geometries for all conformers were those obtained at the MP2/cc-pvdz+S level. $\Delta E$ s are relative to the Trans 2 energies.

$\mathrm{b}$ The results of HF calculations are here reported for comparison

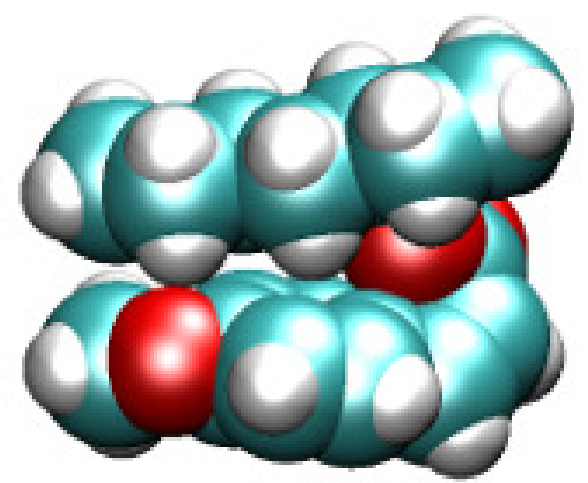

FIG. 3. van-der-Waals representation of the Cis2 isomer optimized at the $\mathrm{mp} 2 / \mathrm{cc}-\mathrm{pvdz}+\mathrm{S}$ level. Atom color codes as in Fig. 1.

The data reported in Tables I and II indicate that the above discussed greater relative stability of Trans 2 with respect to Trans1 and of Cis2 with respect to Cis1 - as well as the considerably lower stability of Cis 3 compared to the other forms, are maintained throughout all the correlated wavefunction method/basis set combinations investigated in this work.

Nonetheless, with the exception of Cis3, a closer inspection of Tables I and II reveals a strong dependence of the (packed)cis-vs-trans relative stability from both basis set and level of theory. At the mp2 level, the larger triple- $\zeta$ basis set favours the Trans 2 isomer. However, by releasing the frozen core approximation at the tzp++ level, the Cis 2 structure becomes more stable than Trans2. Notably, the Cis2 isomer is also favoured at the CCSD+T(CCSD)/dzp++ level of theory (Table II).

An interesting point emerges by inspecting the $\mathrm{CC} / \mathrm{dzp}++$ energies trend (Table II): the Trans 2 is favoured at the CCSD level, while, by including triples, the situation is reversed and the Cis 2 is predicted as the most stable structure. It should be stressed, however, that the energy differences between the two most stable isomers (Cis2 and Trans2) with the larger basis set (dzp++, see Table II) are below $1 \mathrm{kcal} / \mathrm{mol}$. In particular, such energy differences are nearly equal to $\mathrm{kT}$ (at $298 \mathrm{~K}$ ) at the CCSD+T(CCSD)/dzp++ level. In addition, the energy difference between the two most stable isomers (Cis2 and Trans2) calculated at the mp2 level with the largest basis set (tzp++, 
see Table I), is below $1 \mathrm{kcal} / \mathrm{mol}$ with the Frozen Core approximation, and it becomes lower than $\mathrm{kT}$ (at $298 \mathrm{~K}$ ) by including full-core correlation. On the other hand, Cis 3 always remains appreciably less stable than Cis 1 and Cis 2 in all the CC calculations, independently of the chosen basis set. In particular, at the CCSD+T(CCSD)/dzp++ level, Cis3 is higher in energy than Cis 1 and Cis 2 by $4.221 \mathrm{kcal} / \mathrm{mol}$ and $5.143 \mathrm{kcal} / \mathrm{mol}$, respectively. This indicates that, in general, a packed structure of the cis isomer should be favored over the unpacked cis form, as the energy gain associated to intramolecular vdw interactions is greater than $\pi$-conjugation effects in cis-OMC.

Notably, this argument is supported by the comparison of the post-HF results with the packed cis-trans energy differences predicted at the HF level (Table I), which are larger than $10 \mathrm{kcal} / \mathrm{mol}$. Remarkably, at the HF level, that does not account for dispersion, the unpacked Cis 3 structure - characterized by a conjugation extent greater than Cis 1 and Cis 2 is the most stable among the here studied cis conformers, the energy differences with Cis 1 and Cis 2 being $5.868 \mathrm{kcal} / \mathrm{mol}$ and $2.908 \mathrm{kcal} / \mathrm{mol}$, respectively. Hence, dispersion interactions are playing a substantial role in stabilizing the packed cis structures by favouring folded geometries characterized by significant intra-molecular van der Waals interactions between the aliphatic and $\pi$ structures, as shown in Figure 3 for the Cis 2 conformer.

Such a packing (folding), which should likely accompany the trans-cis OMC isomerization, brings about substantial size changes in the molecular shape. These structural changes can be quantitatively estimated by inspecting Table III, where the moments of inertia of the five investigated conformers are reported. Indeed, the moments of inertia along two principal axes nearly double in passing from the packed (Cis1/Cis2) to the unpacked (Trans1/Trans2) isomers, leading to a quite different rotational behaviour. As expected, the moments of inertia of the Cis 3 structure are closer to the values pertaining to the two trans isomers, which have a similar unpacked structure. However, Cis3 represents an energetically unfavored arrangement of cis-OMC with respect to the packed (Cis1/Cis2) forms, which may likely be less visited in the conformational space of this molecule. Also interestingly, we note in passing that the structure of the $T_{1}$ excited triplet state, recently calculated at the $\omega \mathrm{b} 97 \mathrm{xd} / 6-311 \mathrm{~g}(\mathrm{~d}, \mathrm{p})$, level ${ }^{31}$ resembles rather closely the packed arrangement of the Cis1/Cis2 conformers. Hence, the considerable reduction in the moments of inertia in passing from the (unpacked) trans to the (packed) cis geometry might be a clue for explaining the efficient nonradiative heat transfer experimentally associated to the transcis interconversion. ${ }^{30,31}$

Overall, these post-HF results on the structure and energetics of octinoxate conformers - in particular, the comparison with the HF energy differences, have highlighted the relevance of electronic correlation in stabilizing packed geometries of this molecule. To explore whether less CPU-intensive DFT methods could succeed in capturing - at least in part - these subtle correlation effects in the OMC isomers, and whether DFT might be less sensitive to the choice of basis set than post-HF approaches, we performed a series of single point energy calculations on the five MP2/cc-pvdz+S optimized geometries using a selection of (dispersion-corrected) DFTfunctionals. In particular, we examined: i) the pure-GGA PBE functional with PW basis (PBE/PW, used in our preparatory FPMD simulations) with $\mathrm{D} 2^{54}$ and $\mathrm{D} 3 \mathrm{BJ}{ }^{69}$ dispersion; ii) PBE-D3BJ with the cc-pvdz+S basis; iii-iv) the rangeseparated hybrid functionals $\omega \mathrm{b} 97 \mathrm{xd}^{66}$ and LC- $\omega \mathrm{PBE}^{67}$ with selected basis sets; the double hybrid functional B2PLYP ${ }^{68}$ with all basis sets used for the mp2 calculations in Table I. The resulting relative energies (i.e. the energy differences) of the five conformers are reported in table IV.

Surprisingly, all DFT results presented here - independently on their accuracy (functional/basis set combination) and on the presence/absence of dispersion corrections - indicate that the two trans structures are more stable than the cis ones, in line with available DFT literature data. ${ }^{48,50}$

As expected, all DFT functionals without dispersion corrections do not accurately reproduce the relative stability of the packed vs. unpacked conformers, largely overestimating the energy difference between these structure - for example, the Cis2/Trans2 energy difference amounts to 10.671 $\mathrm{kcal} / \mathrm{mol}$ and $7.810 \mathrm{kcal} / \mathrm{mol}$ at the PBE/PW (rung 2) and B2PLYP/tzp++ (rung 5) levels respectively. Such cis-trans energy differences are not much smaller than the corresponding HF values (also reported in table IV), and roughly in keeping with available literature data from DFT calculations without dispersion, which predicted energy differences of $15-16 \mathrm{kcal} / \mathrm{mol}(\mathrm{PBE} 0 / 6-31+\mathrm{g}(2 \mathrm{~d})$, with implicit apolar solvents) ${ }^{48}$ and absolute enthalpy differences of $4.7 \mathrm{kcal} / \mathrm{mol}$ with B3LYP/6-311++G(d,p) at $\mathrm{T}=298 \mathrm{~K}$ in the gas phase. ${ }^{50}$ Interestingly, the DFT functionals without empirical dispersion - including B2PLYP - predict the unpacked system (Cis3) to be the most stable among the three cis conformers in view of its greater conjugation, as also found by the (noncorrelated) HF calculations.

This known limitation of DFT in dealing with vdw interactions is partially cured by the addition of empirical dispersion - for example, the Cis2/Trans2 energy difference is nearly halved by adding to B2PLYP/tzp++ the D3BJ correction, reaching a value of $4.387 \mathrm{kcal} / \mathrm{mol}$. Even the description of vdw interactions with the least accurate approach (pureGGA PBE functional and PW) greatly benefits from inclusion of empirical dispersion, as we already noted in previous work with PBE-D2 ${ }^{84,85}$. Finally, also the results of the range-separated hybrids are nicely improved by the inclusion of dispersion. Indeed, even though the Cis $2 /$ Trans2 energy difference always remains above $2 \mathrm{kcal} / \mathrm{mol}$, the lowest value $(2.318 \mathrm{kcal} / \mathrm{mol})$ is obtained with the range separated LC$\omega$ PBE-D3BJ functional. To the best of our knowledge, no literature data on the cis-trans OMC energy difference with dispersion-corrected DFT functionals are available to compare with our results. Nevertheless, comparison of the data in Table IV with the corresponding post-HF results (Tables I-II) indicates that a fraction of the correlation energy responsible of the vdw intramolecular stabilizing interactions in the packed cis forms is not completely captured by the DFTapproaches here selected.

On the bright side, we notice that, with the only exception of PBE-D3BJ/PW, the relative energy ordering among 
the cis conformers calculated with the dispersion-corrected DFT functionals agrees with the post-HF data presented here. Overall, by considering the lower computational cost of DFT vs post-HF approaches, this result might be considered satisfactory, especially if we keep into account that the energy differences at the CCSD+T(CCSD)/dzp++ level are below 1 $\mathrm{kcal} / \mathrm{mol}$, the graal of precision in quantum chemistry.

\section{CONCLUSIONS}

In this work we have presented a computational analysis on the relative stability of cis- vs trans- OMC (octinoxate), a molecule largely used as component of UV-protecting sunscreen creams. Adopting post-HF level calculations up to the CCSD-T(CCSD) level of theory we could predict that the cis-OMC isomer could be more stable than previously reported, and that its ground state energy is slightly lower $(\approx$ $0.5 \mathrm{kcal} / \mathrm{mol}$ ) than the energy of trans-OMC. Calculations on the same structures with a series of DFT-based approaches (also including the B2PLYP double hybrid functional) combined with empirical dispersion corrections did not succeed in reproducing this subtle and unexpected stabilization of cisOMC. Actually, capturing such a tiny energy difference represents a true challenge for any computational approach.

We could moreover pinpoint the reason of the slightly greater stability of cis-OMC predicted by post-HF calculations: in the cis conformers, the two structural motifs of the molecule - a nearly linear aliphatic chain and a cinnamate backbone - may interact very favourably via van der Waals interactions. This interaction is strong enough to match the loss of $\pi$-conjugation as a consequence of folding. On the other hand, the stability of the trans conformers is likely due to a large $\pi$-conjugation, while the trans (E) arrangement hinders the folding needed to achieve maximum intra-molecular van der Waals stabilization.

With respect to the practical use of OMC as UV filter, the most accurate analyses indicate a co-participation of both isomers to the filtering effects: the trans-OMC (which has a larger $\pi$-conjugation) absorbs light in the $300 \mathrm{~nm}$ region. Then, it converts to cis-OMC (a poorer absorber), which nonradiatively releases heat to the ambient. We might argue that in the trans(unfolded)-to-cis(folded) light-induced conversion, the variation of molecular size (thus, the change in the inertia moments) here highlighted might effectively contribute to the dissipation of the energy accumulated upon light absorption. In perspective, the behaviour, at thermal energy, of the two isomers could be in principle investigated via molecular dynamics simulations to fully capture the differences in their complex rotational characteristics.

\section{DEDICATION}

This paper honors Professor Giorgina Corongiu for her pioneering contributions to quantum chemistry.

\section{SUPPLEMENTARY MATERIAL}

The supplementary material contains five text files with the cartesian coordinates of the MP2/cc-pvdz+S optimized geometries of the five octinoxate conformers considered in this study (Cis1, Trans1, Cis2, Trans2, Cis3).

\section{ACKNOWLEDGMENTS}

Financial support of University of Insubria FAR2019 is gratefully acknowledged. Dr. Cristiano Invernizzi is gratefully acknowledged for useful discussions.

\section{DATA AVAILABILITY STATEMENT}

The data that supports the findings of this study are available within the article and its supplementary material.

\footnotetext{
${ }^{1}$ T. Diepgen and V. Mahler, "The epidemiology of skin cancer," British Journal of Dermatology 146, 1-6 (2002).
}

${ }^{2}$ N. A. Shaath, "Ultraviolet filters," Photochemical and Photobiological Sciences 9, 464-469 (2010).

${ }^{3}$ D. R. Sambandan and D. Ratner, "Sunscreens: An overview and update," Journal of the American Academy of Dermatology 64, 748-758 (2011).

${ }^{4}$ S. Pattanaargson and P. Limphong, "Stability of octyl methoxycinnamate and identification of its photo-degradation product," International Journal of Cosmetic Science 23, 153-160 (2001).

${ }^{5}$ H. Gonzenbach, T. J. Hill, and T. G. Truscott, "The triplet energy levels of uva and uvb sunscreens," Journal of Photochemistry and Photobiology, B: Biology 16, 377-379 (1992).

${ }^{6}$ J. K. Broadbent, B. S. Martincigh, M. W. Raynor, L. F. Salter, R. Moulder, P. Sjöberg, and K. E. Markides, "Capillary supercritical fluid chromatography combined with atmospheric pressure chemical ionisation mass spectrometry for the investigation of photoproduct formation in the sunscreen absorber 2-ethylhexyl-p-methoxycinnamate," Journal of Chromatography A 732, 101-110 (1996).

${ }^{7}$ V. Ambrogi, L. Latterini, F. Marmottini, C. Pagano, and M. Ricci, "Mesoporous silicate $\mathrm{mcm}-41$ as a particulate carrier for octyl methoxycinnamate: Sunscreen release and photostability," Journal of Pharmaceutical Sciences 102, 1468-1475 (2013).

${ }^{8}$ O. I. Parisi, D. Aiello, M. F. Casula, F. Puoci, R. Malivindi, L. Scrivano, and F. Testa, "Mesoporous nanocrystalline tio2 loaded with ferulic acid for sunscreen and photo-protection: Safety and efficacy assessment," RSC Advances 6, 83767-83775 (2016).

${ }^{9}$ N. Serpone, A. Salinaro, A. V. Emeline, S. Horikoshi, H. Hidaka, and J. Zhao, "An in vitro systematic spectroscopic examination of the photostabilities of a random set of commercial sunscreen lotions and their chemical uvb/uva active agents," Photochem. Photobiol. Sci. 1, 970-981 (2002).

${ }^{10}$ S. Pattanaargson, T. Munhapol, P. Hirunsupachot, and P. Luangthongaram, "Photoisomerization of octyl methoxycinnamate," Journal of Photochemistry and Photobiology A: Chemistry 161, 269-274 (2004).

${ }^{11}$ R. M. Sayre, J. C. Dowdy, A. J. Gerwig, W. J. Shields, and R. V. Lloyd, "Unexpected photolysis of the sunscreen octinoxate in the presence of the sunscreen avobenzoneI]," Photochemistry and Photobiology 81, 452 (2005).

${ }^{12}$ L. A. MacManus-Spencer, M. L. Tse, J. L. Klein, and A. E. Kracunas, "Aqueous photolysis of the organic ultraviolet filter chemical octyl methoxycinnamate," Environmental Science and Technology 45, 39313937 (2011).

${ }^{13}$ S. P. Huong, V. Andrieu, J. P. Reynier, E. Rocher, and J. D. Fourneron, "The photoisomerization of the sunscreen ethylhexyl p-methoxy cinnamate and its influence on the sun protection factor," Journal of Photochemistry and Photobiology A: Chemistry 186, 65-70 (2007).

${ }^{14}$ K. M. Hanson, S. Narayanan, V. M. Nichols, and C. J. Bardeen, "Photochemical degradation of the uv filter octyl methoxycinnamate in solution 
and in aggregates," Photochemical and Photobiological Sciences 14, 1607 $1616(2015)$

${ }^{15}$ T. M. Karpkird, S. Wanichweacharungruang, and B. Albinsson, "Photophysical characterization of cinnamates," Photochemical and Photobiological Sciences 8, 1455-1460 (2009).

${ }^{16}$ A. J. M. Santos, M. S. Miranda, and J. C. E. da Silva, "The degradation products of uv filters in aqueous and chlorinated aqueous solutions," Water Research 46, 3167-3176 (2012).

${ }^{17}$ C. Puglia, F. Bonina, L. Rizza, P. Blasi, A. Schoubben, R. Perrotta, M. S. Tarico, and E. Damiani, "Lipid nanoparticles as carrier for octylmethoxycinnamate: In vitro percutaneous absorption and photostability studies," Journal of Pharmaceutical Sciences 101, 301-311 (2012).

${ }^{18}$ A. L. M. Daneluti, F. M. Neto, M. V. R. Velasco, A. R. Baby, and J. do Rosário Matos, "Evaluation and characterization of the encapsulation/entrapping process of octyl methoxycinnamate in ordered mesoporous silica type sba-15," Journal of Thermal Analysis and Calorimetry 131, 789798 (2018).

${ }^{19}$ R. Rodil, M. Moeder, R. Altenburger, and M. Schmitt-Jansen, "Photostability and phytotoxicity of selected sunscreen agents and their degradation mixtures in water," Analytical and Bioanalytical Chemistry 395, 15131524 (2009).

${ }^{20}$ D. Dondi, A. Albini, and N. Serpone, "Interactions between different solar uvb/uva filters contained in commercial suncreams and consequent loss of uv protection," Photochemical and Photobiological Sciences 5, 835-843 (2006).

${ }^{21}$ S. Scalia and M. Mezzena, "Photostabilization effect of quercetin on the uv filter combination, butyl methoxydibenzoylmethane-octyl methoxycinnamate," Photochemistry and Photobiology 86, 273-278 (2010).

${ }^{22}$ L. A. Baker, S. E. Greenough, and V. G. Stavros, "A perspective on the ultrafast photochemistry of solution-phase sunscreen molecules," Journal of Physical Chemistry Letters 7, 4655-4665 (2016).

${ }^{23}$ E. L. Holt and V. G. Stavros, "Applications of ultrafast spectroscopy to sunscreen development, from first principles to complex mixtures," International Reviews in Physical Chemistry 38, 243-285 (2019).

${ }^{24}$ K. M. Krokidi, M. A. P. Turner, P. A. J. Pearcy, and V. G. Stavros, "A systematic approach to methyl cinnamate photodynamics," Molecular Physics 119, e1811910 (2021).

${ }^{25}$ J. M. Woolley, R. Losantos, D. Sampedro, and V. G. Stavros, "Computational and experimental characterization of novel ultraviolet filters," Physical Chemistry Chemical Physics 22, 25390-25395 (2020).

${ }^{26}$ S. Kenjo, Y. Iida, N. Chaki, S. nosuke Kinoshita, Y. Inokuchi, K. Yamazaki, and T. Ebata, "Laser spectroscopic study on sinapic acid and its hydrated complex in a cold gas phase molecular beam," Chemical Physics 515, 381386 (2018).

${ }^{27}$ E. M. Tan, M. Hilbers, and W. J. Buma, "Excited-state dynamics of isolated and microsolvated cinnamate-based uv-b sunscreens," Journal of Physical Chemistry Letters 5, 2464-2468 (2014).

${ }^{28}$ Y. Miyazaki, K. Yamamoto, J. Aoki, T. Ikeda, Y. Inokuchi, M. Ehara, and T. Ebata, "Experimental and theoretical study on the excited-state dynamics of ortho-,meta-, and para-methoxy methylcinnamate," Journal of Chemical Physics 141, 244313 (2014)

${ }^{29}$ Y. Miyazaki, Y. Inokuchi, N. Akai, and T. Ebata, "Direct spectroscopic evidence of photoisomerization in para -methoxy methylcinnamate revealed by low-temperature matrix-isolation ftir spectroscopy," Journal of Physical Chemistry Letters 6, 1134-1139 (2015).

${ }^{30}$ Y. Peperstraete, M. Staniforth, L. A. Baker, N. D. Rodrigues, N. C. ColeFilipiak, W. D. Quan, and V. G. Stavros, "Bottom-up excited state dynamics of two cinnamate-based sunscreen filter molecules," Physical Chemistry Chemical Physics 18, 28140-28149 (2016).

${ }^{31}$ S. Muramatsu, S. Nakayama, S. N. Kinoshita, Y. Onitsuka, H. Kohguchi, Y. Inokuchi, C. Zhu, and T. Ebata, "Electronic state and photophysics of 2-ethylhexyl-4-methoxycinnamate as uv-b sunscreen under jet-cooled condition," Journal of Physical Chemistry A 124, 1272-1278 (2020).

${ }^{32}$ M. Promkatkaew, S. Suramitr, T. M. Karpkird, S. Namuangruk, M. Ehara, and S. Hannongbua, "Absorption and emission spectra of ultraviolet b blocking methoxy substituted cinnamates investigated using the symmetryadapted cluster configuration interaction method," Journal of Chemical Physics 131, 224306 (2009).

${ }^{33}$ M. D. Groot, E. V. Gromov, H. Köppel, and W. J. Buma, "High-resolution spectroscopy of methyl 4-hydroxycinnamate and its hydrogen-bonded wa- ter complex," Journal of Physical Chemistry B 112, 4427-4434 (2008).

${ }^{34}$ D. Sampedro, "Computational exploration of natural sunscreens," Physical Chemistry Chemical Physics 13, 5584-5586 (2011).

${ }^{35}$ M. Promkatkaew, S. Suramitr, T. Karpkird, M. Ehara, and S. Hannongbua, "Absorption and emission properties of various substituted cinnamic acids and cinnamates, based on tddft investigation," 113, 542-554 (2013).

${ }^{36}$ E. M. M. Tan, S. Amirjalayer, S. Smolarek, A. Vdovin, A. M. Rijs, and W. J. Buma, "Conformational heterogeneity of methyl 4hydroxycinnamate: A gas-phase uv-ir spectroscopic study," The Journal of Physical Chemistry B 117, 4798-4805 (2013), pMID: 23574393, https://doi.org/10.1021/jp312624e.

${ }^{37}$ X. P. Chang, C. X. Li, B. B. Xie, and G. Cui, "Photoprotection mechanism of p-methoxy methylcinnamate: A caspt2 study," Journal of Physical Chemistry A 119, 11488-11497 (2015).

${ }^{38}$ J. Moon, H. Baek, J. S. Lim, and J. Kim, "Tddft and ms-caspt2 study of the excited states of $<i>$ para $</ i>$-methoxymethylcinnamate," Bulletin of the Korean Chemical Society 39, 427-434 (2018).

${ }^{39}$ C. X. Li, W. W. Guo, B. B. Xie, and G. Cui, "Photodynamics of oxybenzone sunscreen: Nonadiabatic dynamics simulations," Journal of Chemical Physics 145, 074308 (2016)

${ }^{40}$ K. Yamazaki, Y. Miyazaki, Y. Harabuchi, T. Taketsugu, S. Maeda, Y. Inokuchi, S. N. Kinoshita, M. Sumida, Y. Onitsuka, H. Kohguchi, M. Ehara, and T. Ebata, "Multistep intersystem crossing pathways in cinnamate-based uv-b sunscreens," Journal of Physical Chemistry Letters 7, 4001-4007 (2016).

${ }^{41}$ X. Zhao, J. Luo, S. Yang, and K. Han, "New insight into the photoprotection mechanism of plant sunscreens: Adiabatic relaxation competing with nonadiabatic relaxation in the cis $\rightarrow$ trans photoisomerization of methyl sinapate," The Journal of Physical Chemistry Letters 10, 4197-4202 (2019), pMID: 31287701, https://doi.org/10.1021/acs.jpclett.9b01651.

${ }^{42}$ S. nosuke Kinoshita, Y. Harabuchi, Y. Inokuchi, S. Maeda, M. Ehara, K. Yamazaki, and T. Ebata, "Substitution effect on the nonradiative decay and trans $\rightarrow$ cis photoisomerization route: a guideline to develop efficient cinnamate based sunscreens," Physical Chemistry Chemical Physics (2020), $10.1039 / \mathrm{d} 0 \mathrm{cp} 04402 \mathrm{~d}$.

${ }^{43}$ J. Fan, W. Roeterdink, and W. J. Buma, "Excited-state dynamics of isolated and (micro)solvated methyl sinapate: the bright and shady sides of a natural sunscreen," Molecular Physics 119, e1825850 (2021).

${ }^{44}$ X.-Y. Xie, C.-X. Li, Q. Fang, and G. Cui, "Mechanistic photochemistry of methyl-4-hydroxycinnamate chromophore and its onewater complexes: Insights from ms-caspt2 study," The Journal of Physical Chemistry A 120, 6014-6022 (2016), pMID: 27398611, https://doi.org/10.1021/acs.jpca.6b05899.

${ }^{45}$ S. N. Kinoshita, Y. Inokuchi, Y. Onitsuka, H. Kohguchi, N. Akai, T. Shiraogawa, M. Ehara, K. Yamazaki, Y. Harabuchi, S. Maeda, and T. Ebata, "The direct observation of the doorway $1 \mathrm{n}$ state of methylcinnamate and hydrogen-bonding effects on the photochemistry of cinnamate-based sunscreens," Physical Chemistry Chemical Physics 21, 19755-19763 (2019).

${ }^{46}$ X. Zhao, F. Ji, Y. Liang, P. Li, Y. Jia, X. Feng, Y. Sun, Y. Shi, L. Zhu, and G. Zhao, "Theoretical and spectroscopic investigation on ultrafast nonadiabatic photoprotective mechanism of novel ultraviolet protective compounds inspired by natural sunscreens," Journal of Luminescence 223, 117228 (2020).

${ }^{47}$ M. D. Horbury, A. L. Flourat, S. E. Greenough, F. Allais, and V. G. Stavros, "Investigating isomer specific photoprotection in a model plant sunscreen," Chem. Commun. 54, 936-939 (2018).

${ }^{48}$ L. F. Alves, R. Gargano, S. K. Alcanfor, L. A. Romeiro, and J. B. Martins, "A chromophoric study of 2-ethylhexyl p-methoxycinnamate," Chemical Physics Letters 516, 162-165 (2011).

${ }^{49}$ A. Gackowska, M. Przybyłek, W. Studziński, and J. Gaca, "Experimental and theoretical studies on the photodegradation of 2-ethylhexyl 4-methoxycinnamate in the presence of reactive oxygen and chlorine species," Central European Journal of Chemistry 12, 612-623 (2014).

${ }^{50}$ M. S. Miranda, L. P. da Silva, and J. C. G. E. da Silva, "Uv filter 2ethylhexyl 4-methoxycinnamate: a structure, energetic and uv-vis spectral analysis based on density functional theory," Journal of Physical Organic Chemistry 27, 47-56 (2014).

${ }^{51}$ R. D. Garcia, V. G. Maltarollo, K. M. Honório, and G. H. Trossini, "Benchmark studies of uv-vis spectra simulation for cinnamates with uv filter profile," Journal of Molecular Modeling 21, 1-13 (2015). 
${ }^{52}$ R. Car and M. Parrinello, "Unified approach for molecular dynamics and density-functional theory," Phys. Rev. Lett. 55, 2471-2474 (1985).

${ }^{53}$ J. P. Perdew, K. Burke, and M. Ernzerhof, "Generalized gradient approximation made simple,” Phys. Rev. Lett. 77, 3865-3868 (1996).

${ }^{54}$ S. Grimme, "Semiempirical gga-type density functional constructed with a long-range dispersion correction," Journal of Computational Chemistry 27, 1787-1799 (2006), https://onlinelibrary.wiley.com/doi/pdf/10.1002/jcc.20495.

${ }^{55} \mathrm{C}$. Møller and M. S. Plesset, "Note on an approximation treatment for many-electron systems," Physical Review 46, 618-622 (1934).

${ }^{56}$ T. H. Dunning and P. J. Hay, "Gaussian basis sets for molecular calculations," Methods of Electronic Structure Theory , 1-27 (1977).

${ }^{57}$ T. H. Dunning, "Gaussian basis sets for use in correlated molecular calculations. i. the atoms boron through neon and hydrogen," The Journal of Chemical Physics 90, 1007-1023 (1989).

${ }^{58}$ P. L. Barbieri, P. A. Fantin, and F. E. Jorge, "Gaussian basis sets of triple and quadruple zeta valence quality for correlated wave functions," Molecular Physics 104, 2945-2954 (2006).

${ }^{59}$ P. A. Fantin, P. L. Barbieri, A. C. Neto, and F. E. Jorge, "Augmented gaussian basis sets of triple and quadruple zeta valence quality for the atoms $\mathrm{h}$ and from li to ar: Applications in hf, mp2, and dft calculations of molecular dipole moment and dipole (hyper)polarizability," Journal of Molecular Structure: THEOCHEM 810, 103-111 (2007).

${ }^{60} \mathrm{~J}$. Ĉízek, "On the correlation problem in atomic and molecular systems. calculation of wavefunction components in ursell-type expansion using quantum-field theoretical methods," The Journal of Chemical Physics 45, 4256-4266 (1966).

${ }^{61}$ J. Čižek and J. Paldus, "Correlation problems in atomic and molecular systems iii. rederivation of the coupled-pair many-electron theory using the traditional quantum chemical methodst," International Journal of Quantum Chemistry 5, 359-379 (1971).

${ }^{62}$ G. D. Purvis and R. J. Bartlett, "A full coupled-cluster singles and doubles model: The inclusion of disconnected triples," The Journal of Chemical Physics 76, 1910-1918 (1982).

${ }^{63}$ K. Raghavachari, G. W. Trucks, J. A. Pople, and M. Head-Gordon, "A fifthorder perturbation comparison of electron correlation theories," Chemical Physics Letters 157, 479-483 (1989).

${ }^{64}$ Y. S. Lee, S. A. Kucharski, and R. J. Bartlett, "A coupled cluster approach with triple excitations," The Journal of Chemical Physics 81, 5906-5912 (1984).

${ }^{65}$ J. P. Perdew, A. Ruzsinszky, J. Tao, V. N. Staroverov, G. E. Scuseria, and G. I. Csonka, "Prescription for the design and selection of density functional approximations: More constraint satisfaction with fewer fits," The Journal of Chemical Physics 123, 062201 (2005), https://doi.org/10.1063/1.1904565.

${ }^{66}$ J.-D. Chai and M. Head-Gordon, "Long-range corrected hybrid density functionals with damped atom-atom dispersion corrections," Phys. Chem. Chem. Phys. 10, 6615-6620 (2008).

${ }^{67}$ O. A. Vydrov and G. E. Scuseria, "Assessment of a long-range corrected hybrid functional," The Journal of Chemical Physics 125, 234109 (2006), https://doi.org/10.1063/1.2409292.

${ }^{68} \mathrm{~S}$. Grimme, "Semiempirical hybrid density functional with perturbative second-order correlation," Journal of Chemical Physics 124, 034108 (2006).

${ }^{69} \mathrm{~S}$. Grimme, S. Ehrlich, and L. Goerigk, "Effect of the damping function in dispersion corrected density functional theory," Journal of Computational Chemistry 32, 1456-1465 (2011).

70"Car-parrinello molecular dynamics cpmd code," Copyright IBM Corp. 1990-2019, MPI für Festkörperforschung Stuttgart, 1997-2001.

${ }^{71}$ T. D. Kühne, M. Iannuzzi, M. D. Ben, V. V. Rybkin, P. Seewald, F. Stein, T. Laino, R. Z. Khaliullin, O. Schütt, F. Schiffmann, D. Golze, J. Wilhelm, S. Chulkov, M. H. Bani-Hashemian, V. Weber, U. Borštnik, M. Taillefumier, A. S. Jakobovits, A. Lazzaro, H. Pabst, T. Müller, R. Schade, M. Guidon, S. Andermatt, N. Holmberg, G. K. Schenter, A. Hehn, A. Bussy, F. Belleflamme, G. Tabacchi, A. Glöß, M. Lass, I. Bethune, C. J. Mundy, C. Plessl, M. Watkins, J. VandeVondele, M. Krack, and J. Hutter, "Cp2k: An electronic structure and molecular dynamics software package quickstep: Efficient and accurate electronic structure calculations," Journal of Chemical Physics 152, 194103 (2020).
${ }^{72}$ X. Zhou, T. A. Wesolowski, G. Tabacchi, E. Fois, G. Calzaferri, and A. Devaux, "First-principles simulation of the absorption bands of fluorenone in zeolite 1," Phys. Chem. Chem. Phys. 15, 159-167 (2013).

${ }^{73}$ R. Arletti, E. Fois, L. Gigli, G. Vezzalini, S. Quartieri, and G. Tabacchi, "Irreversible conversion of a water-ethanol solution into an organized twodimensional network of alternating supramolecular units in a hydrophobic zeolite under pressure," Angewandte Chemie International Edition 56, 2105-2109 (2017).

${ }^{74} \mathrm{G}$. Tabacchi, "Supramolecular organization in confined nanospaces," ChemPhysChem 19, 1249-1297 (2018).

${ }^{75}$ E. Fois, G. Tabacchi, and G. Calzaferri, "Orientation and order of xanthene dyes in the one-dimensional channels of zeolite 1: Bridging the gap between experimental data and molecular behavior," Journal of Physical Chemistry C 116, 16784-16799 (2012).

${ }^{76}$ G. Tabacchi, E. Fois, and G. Calzaferri, "Structure of nanochannel entrances in stopcock-functionalized zeolitel composites," Angewandte Chemie International Edition 54, 11112-11116 (2015), https://onlinelibrary.wiley.com/doi/pdf/10.1002/anie.201504745.

${ }^{77}$ G. Tabacchi, G. Calzaferri, and E. Fois, "One-dimensional self-assembly of perylene-diimide dyes by unidirectional transit of zeolite channel openings," Chem. Commun. 52, 11195-11198 (2016).

${ }^{78}$ G. J. Martyna and M. E. Tuckerman, "A reciprocal space based method for treating long range interactions in ab initio and force-field-based calculations in clusters," The Journal of Chemical Physics 110, 2810-2821 (1999), https://doi.org/10.1063/1.477923.

${ }^{79}$ M. J. Frisch, G. W. Trucks, H. B. Schlegel, G. E. Scuseria, M. A. Robb, J. R. Cheeseman, G. Scalmani, V. Barone, B. Mennucci, G. A. Petersson, H. Nakatsuji, M. Caricato, X. Li, H. P. Hratchian, A. F. Izmaylov, J. Bloino, G. Zheng, J. L. Sonnenberg, M. Hada, M. Ehara, K. Toyota, R. Fukuda, J. Hasegawa, M. Ishida, T. Nakajima, Y. Honda, O. Kitao, H. Nakai, T. Vreven, J. A. Montgomery, Jr., J. E. Peralta, F. Ogliaro, M. Bearpark, J. J. Heyd, E. Brothers, K. N. Kudin, V. N. Staroverov, R. Kobayashi, J. Normand, K. Raghavachari, A. Rendell, J. C. Burant, S. S. Iyengar, J. Tomasi, M. Cossi, N. Rega, J. M. Millam, M. Klene, J. E. Knox, J. B. Cross, V. Bakken, C. Adamo, J. Jaramillo, R. Gomperts, R. E. Stratmann, O. Yazyev, A. J. Austin, R. Cammi, C. Pomelli, J. W. Ochterski, R. L. Martin, K. Morokuma, V. G. Zakrzewski, G. A. Voth, P. Salvador, J. J. Dannenberg, S. Dapprich, A. D. Daniels, Farkas, J. B. Foresman, J. V. Ortiz, J. Cioslowski, and D. J. Fox, "Gaussian09 Revision E.01," Gaussian Inc. Wallingford CT 2009.

${ }^{80}$ M. Valiev, E. J. Bylaska, N. Govind, K. Kowalski, T. P. Straatsma, H. J. V. Dam, D. Wang, J. Nieplocha, E. Apra, T. L. Windus, and W. A. D. Jong, "Nwchem: A comprehensive and scalable open-source solution for large scale molecular simulations," Computer Physics Communications 181, 1477-1489 (2010).

${ }^{81}$ E. Aprà, E. J. Bylaska, W. A. D. Jong, N. Govind, K. Kowalski, T. P. Straatsma, M. Valiev, H. J. V. Dam, Y. Alexeev, J. Anchell, V. Anisimov, F. W. Aquino, R. Atta-Fynn, J. Autschbach, N. P. Bauman, J. C. Becca, D. E. Bernholdt, K. Bhaskaran-Nair, S. Bogatko, P. Borowski, J. Boschen, J. Brabec, A. Bruner, E. Cauët, Y. Chen, G. N. Chuev, C. J. Cramer, J. Daily, M. J. Deegan, T. H. Dunning, M. Dupuis, K. G. Dyall, G. I. Fann, S. A. Fischer, A. Fonari, H. Früchtl, L. Gagliardi, J. Garza, N. Gawande, S. Ghosh, K. Glaesemann, A. W. Götz, J. Hammond, V. Helms, E. D. Hermes, K. Hirao, S. Hirata, M. Jacquelin, L. Jensen, B. G. Johnson, H. Jónsson, R. A. Kendall, M. Klemm, R. Kobayashi, V. Konkov, S. Krishnamoorthy, M. Krishnan, Z. Lin, R. D. Lins, R. J. Littlefield, A. J. Logsdail, K. Lopata, W. Ma, A. V. Marenich, J. M. D. Campo, D. Mejia-Rodriguez, J. E. Moore, J. M. Mullin, T. Nakajima, D. R. Nascimento, J. A. Nichols, P. J. Nichols, J. Nieplocha, A. Otero-De-La-Roza, B. Palmer, A. Panyala, T. Pirojsirikul, B. Peng, R. Peverati, J. Pittner, L. Pollack, R. M. Richard, P. Sadayappan, G. C. Schatz, W. A. Shelton, D. W. Silverstein, D. M. Smith, T. A. Soares, D. Song, M. Swart, H. L. Taylor, G. S. Thomas, V. Tipparaju, D. G. Truhlar, K. Tsemekhman, T. V. Voorhis, A. Vázquez-Mayagoitia, P. Verma, O. Villa, A. Vishnu, K. D. Vogiatzis, D. Wang, J. H. Weare, M. J. Williamson, T. L. Windus, K. Woliński, A. T. Wong, Q. Wu, C. Yang, Q. Yu, M. Zacharias, Z. Zhang, Y. Zhao, and R. J. Harrison, "Nwchem: Past, present, and future," Journal of Chemical Physics 152, 184102 (2020).

${ }^{82}$ B. P. Pritchard, D. Altarawy, B. Didier, T. D. Gibson, and T. L. Windus, "New basis set exchange: An open, up-to-date resource for the molecular sciences community," Journal of Chemical Information and Modeling 59, 
4814-4820 (2019).

${ }^{83}$ V. Arjunan, R. Anitha, S. Thenmozhi, M. K. Marchewka, and S. Mohan, "Potential energy profile, structural, vibrational and reactivity descriptors of trans-2-methoxycinnamic acid by ftir, ft-raman and quantum chemical studies," Journal of Molecular Structure 1113, 42-54 (2016).

${ }^{84}$ L. Gigli, R. Arletti, G. Tabacchi, E. Fois, J. G. Vitillo, G. Martra, G. Agostini, S. Quartieri, and G. Vezzalini, "Close-packed dye molecules in zeolite channels self-assemble into supramolecular nanoladders," The Journal of Physical Chemistry C 118, 15732-15743 (2014).
${ }^{85}$ M. Fabbiani, G. Confalonieri, S. Morandi, R. Arletti, S. Quartieri, M. Santoro, F. Di Renzo, J. Haines, R. Fantini, G. Tabacchi, E. Fois, G. Vezzalini, G. Ricchiardi, and G. Martra, "Steering polymer growth by molding nanochannels: 1,5-hexadiene polymerization in high silica mordenite," Microporous and Mesoporous Materials 311, 110728 (2021).

${ }^{86}$ L. Gigli, R. Arletti, G. Tabacchi, M. Fabbiani, J. G. Vitillo, G. Martra, A. Devaux, I. Miletto, S. Quartieri, G. Calzaferri, and E. Fois, "Structure and host-guest interactions of perylene-diimide dyes in zeolite 1 nanochannels," The Journal of Physical Chemistry C 122, 3401-3418 (2018).

${ }^{87}$ D. G. Smith, L. A. Burns, K. Patkowski, and C. D. Sherrill, "Revised damping parameters for the $\mathrm{d} 3$ dispersion correction to density functional theory," Journal of Physical Chemistry Letters 7, 2197-2203 (2016). 